\title{
Knowledge Organization and the 2013 UDC Seminar: An Editorial
}

\author{
Jihee Beak, Inkyung Choi, Sukwon Lee, Hyounjoo Park, \\ Laura Ridenour and Richard P. Smiraglia \\ Information Organization Research Group, School of Information Studies, \\ University of Wisconsin, Milwaukee, Northwest Quad Building B, 2025 E Newport Street, \\ Milwaukee, WI 53211, USA, smiragli@uwm.edu
}

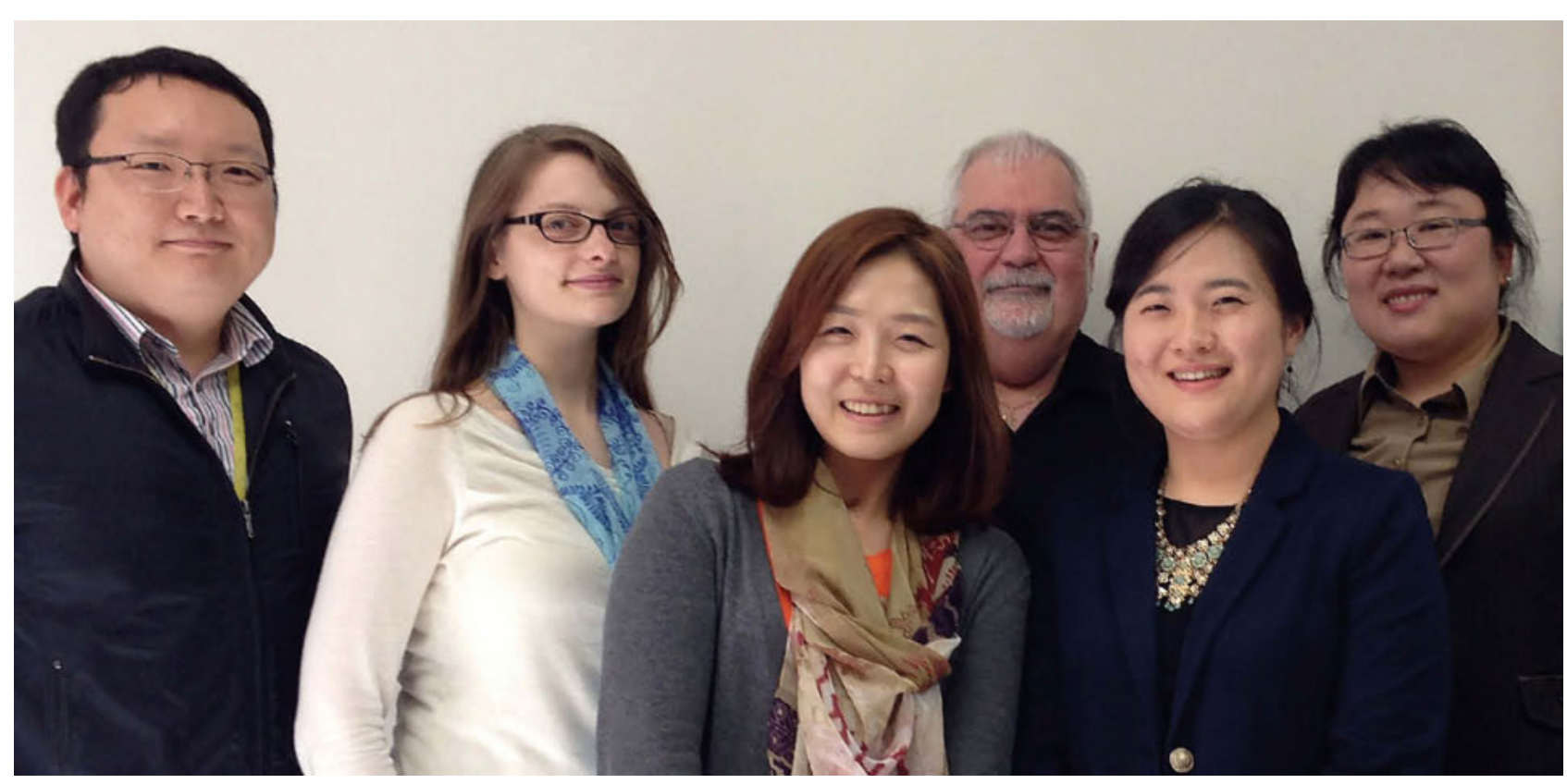

Jihee Beak, Inkyung Choi, Sukwon Lee, Hyounjoo Park, Laura Ridenour and Richard P. Smiraglia. Knowledge Organization and the 2013 UDC Seminar: An Editorial. Knowledge Organization. 42(3), 191-194. 2 references.

The fourth in a series of biennial international UDC seminars was held at the Koninklijke Bibliotheek in The Hague, on 24-25 October 2013. The 2013 seminar was titled "Classification \& Visualization: Interfaces to Knowledge" and was a lively and well-attended two day conference bringing together an international group of researchers unified by the UDC. Our research group was interested in analyzing the domain of the conference, as it were, to see how it might be similar to the broader domain of knowledge organization. To that end we present here a few informetrics and some visualizations of the parameters of the conference.

\subsection{Some domain metrics}

There were twenty-five papers in the conference program, nineteen of which appeared in the printed proceedings. There were forty-three authors of the twenty-five papers, which meant that there were a significant number of collaborations. The mean number of authors per paper was 2.2, and the range was from 1 to 4 . There were 407 citations in the nineteen papers in the published proceedings. The mean number of citations was 21.4 per paper, with a range from 3 to 58 . The authors of the papers came from eleven countries, with the majority from the 


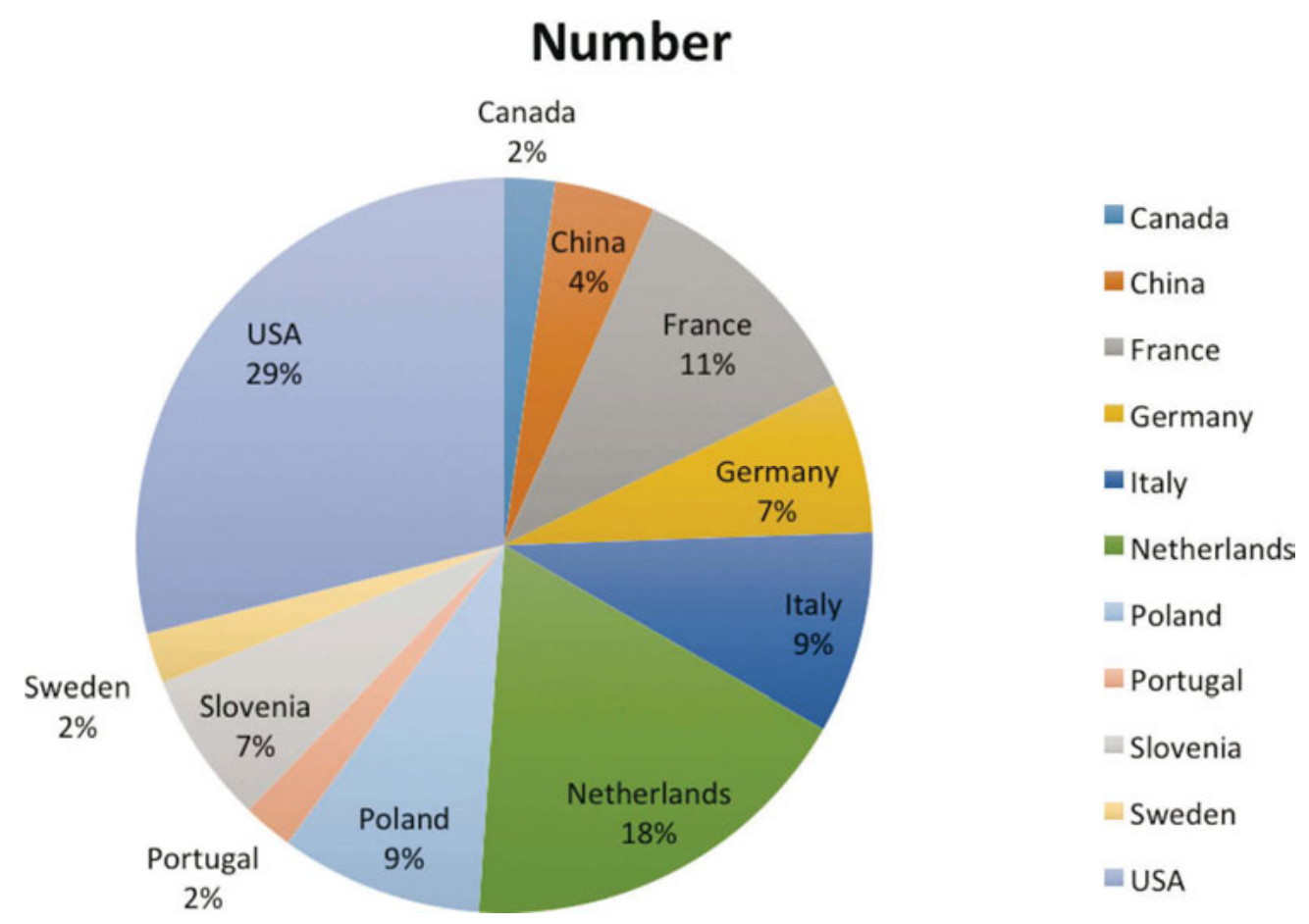

Figure 1. Nationalities of contributing authors

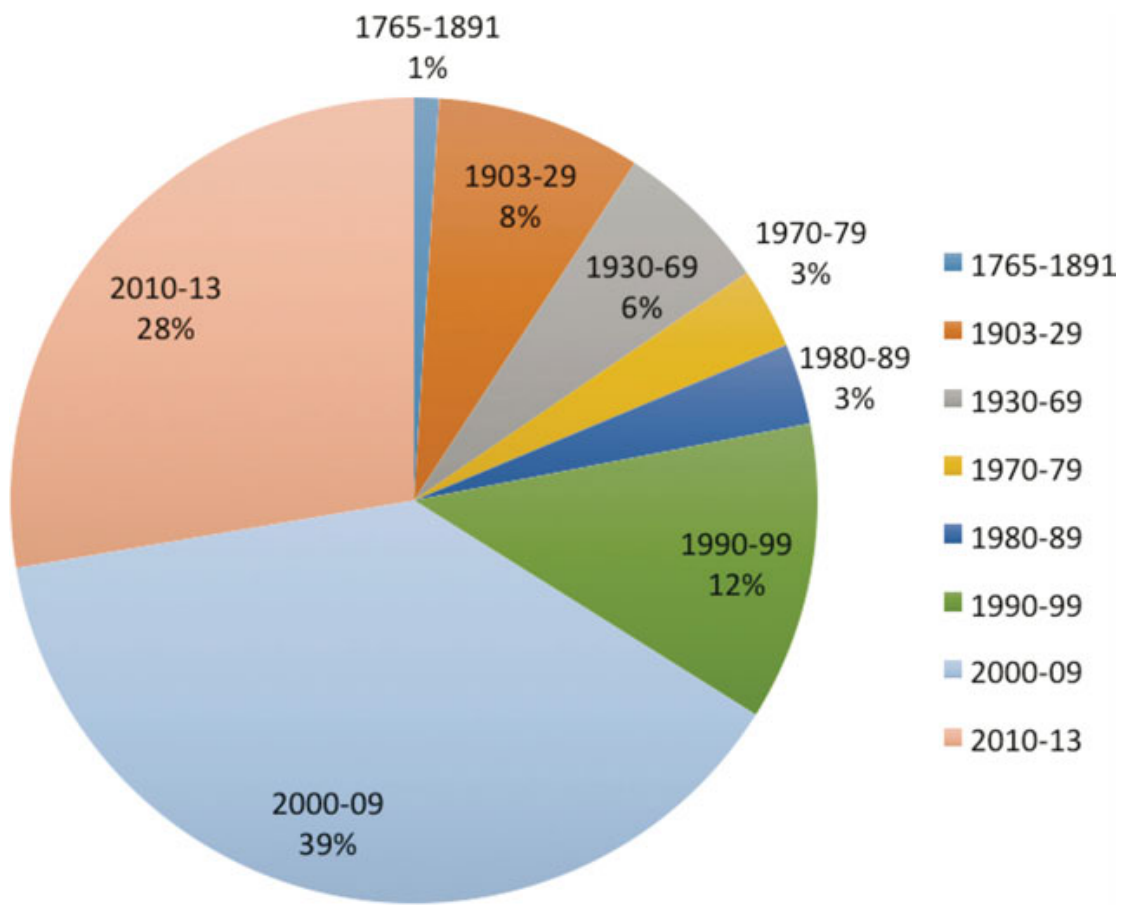

Figure 2. Year of works cited

USA and the Netherlands. Countries of affiliation are visualized in Figure 1.

The year of cited work ranged from 1715 to 2013 . Figure 2 is a visualization of the distribution. A clear majority of citations were to works from 2006 to 2013; in fact there was a dramatic shift in the frequency distribution from 3 to 6 per year up through 2005, to 23 or more in 2006 and beyond; so that tells us that most cited works were published in 2006 or later. In other words, most of the research cited here took place in that past eight years. 


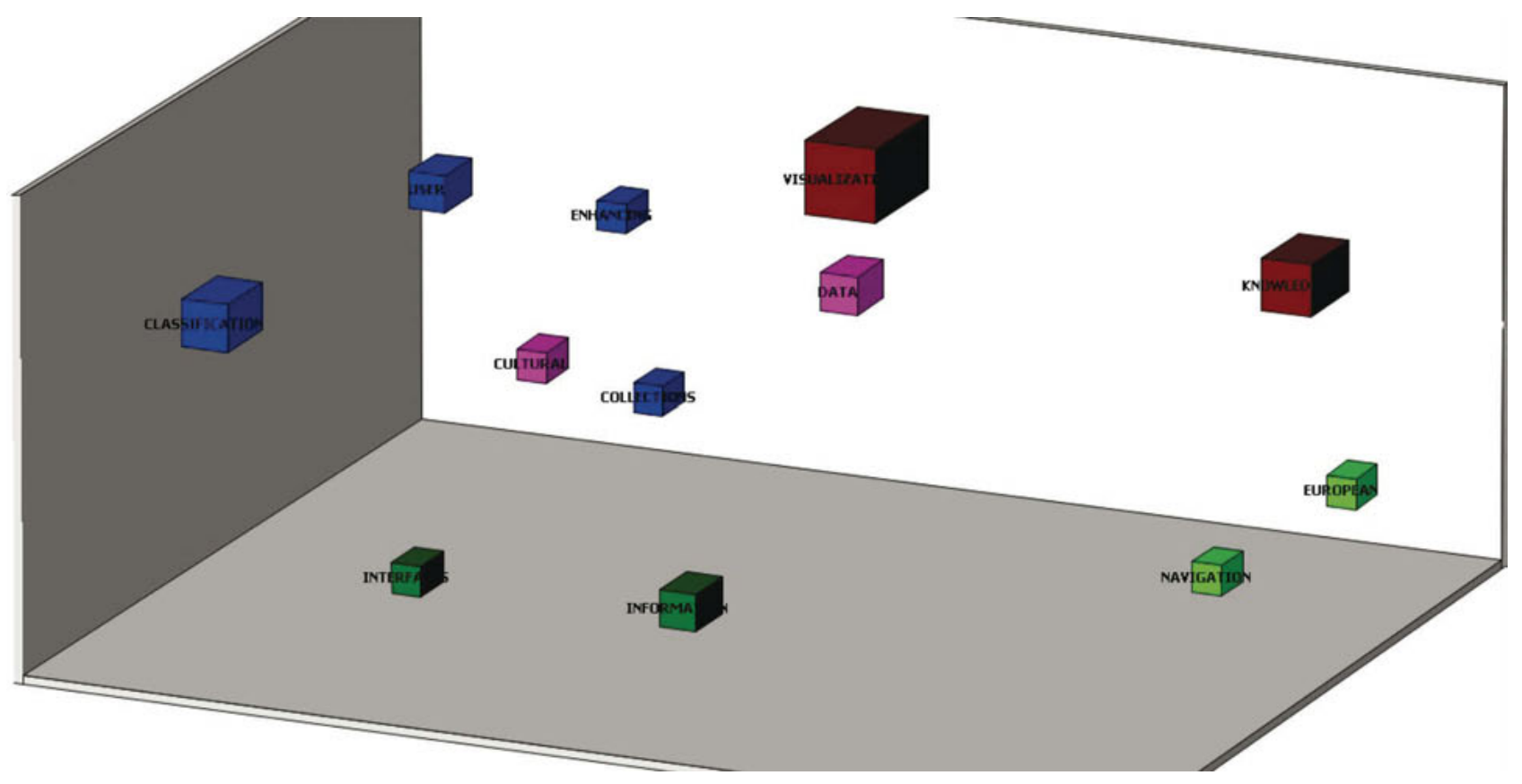

Figure 3. MDS plot of keywords in paper titles.

We entered the titles of the twenty-five papers into WordStat ${ }^{\mathrm{TM}}$ software and created a multi-dimensionally scaled plot of co-occurrence of the most frequently occurring terms; this is a standard technique for visualizing the thematic breadth of a domain. The plot is shown in Figure 3.

What we see is that "visualization," the conference theme, is the anchoring largest cube, with knowledge in second place but at some distance. We see "classification," "cultural" and "European" as key terms from the top of the frequency distribution. The distance among the plotted terms shows there is coherence to the conference theme but not much inherence among the key terms in the titles of the papers. It just means an extension of knowledge visualization is backed by a rather looselydefined intension. A network visualization was created using terms in the twenty-five abstracts, which were plotted using Sci2. This visualization appears in Figure 4.

The largest nodes in the foreground are the same as those in the MDS plot- "Visual" "Inform" "User" „Knowledg,"- - but we also see emergent nodes such as "Design," "Data," “Approach," "Scheme," “Thesaurus," etc. It is testimony to the richness of the domain of this conference that so many terms rise to the top levels of network nodes.

\subsection{Some concluding thoughts}

We were interested in learning whether the domain parameters of this conference were similar to those of knowl- edge organization $(\mathrm{KO})$ as a community. We were able to use comparative data from two earlier studies by Smiraglia (2012, 2013). Concerning collaboration, we see here the usual range that we see in $\mathrm{KO}$ from teams on one side to humanistic approaches on the other side, the mean is low, tending toward the humanistic. There always is constructive tension in $\mathrm{KO}$ between humanist epistemologies (which tend to be historicist and rationalist) and empiricist (which are positivist, quantitative or qualitative). Similarly, concerning numbers of citations and age of cited works, we see a range from a few recent citations, characteristic of scientific epistemologies, to many older citations, characteristic of humanistic epistemologies. This is consistent with the constructive tension in the domain.

The geographic distribution is strikingly different, and here we have to take into account both the venue of the conference and the close adherence to the UDC. The influence of scholarship from The Netherlands is marked and impressive. The fact that most non-USA papers come from Western Europe also is remarkable. This is different from the recent results of $\mathrm{KO}$ in general, in which Brazil has been taking a lead.

The thematic results are fascinating and not particularly surprising. The conference was built around one of the world's leading classifications of knowledge, and the theme was a cohering factor among the papers. On the other hand, the granularity that is visible in the network map shows the richness of the research domain. The UDC community has the potential to lead the evolution of $\mathrm{KO}$ as a research domain. 


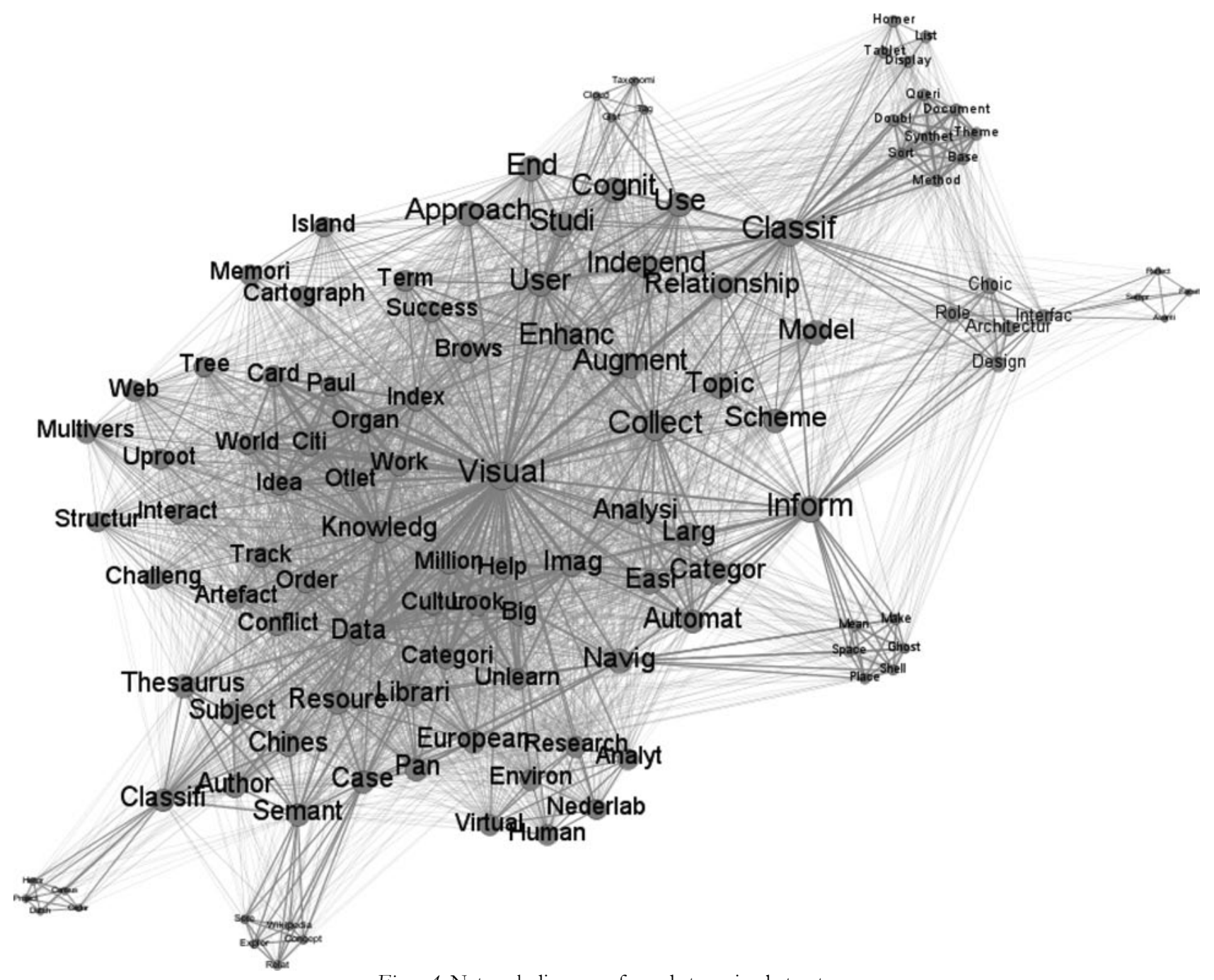

Figure 4. Network diagram of word stems in abstracts.

\section{References}

Smiraglia, Richard P. 2012. Universes, dimensions, domains, intensions and extensions: knowledge organization for the 21 st century. In A. Neelameghan and K.S. Raghavan eds. Categories, contexts, and relations in knowledge organization: Proceedings of the Twelfth International ISKO Conference, 6-9 August 2012, Mysore, India. Advances in knowledge organization 13. Würzburg: Ergon Verlag, 2012, pp. 1-7.

Smiraglia, Richard P. 2013. The epistemological dimension of knowledge organization. IRIS Revista de Informação, Memória e Tecnologia. Forthcoming. 